\title{
ARQUITETURA DE REDUNDÂNCIA DE GATEWAYS IOT EM REDES ZIGBEE
}

\author{
Paulo T. G. Mascarenhas, Francisco Lopes de Caldas Filho, Cássio F. C. Ribeiro, \\ Lucas M. C. e Martins, Pedro H. L. da Costa e Rafael Timóteo de Sousa Júnior \\ Laboratório LATITUDE, Departamento de Engenharia Elétrica, Universidade de Brasília (UnB), Brasília-DF, Brasil
}

\begin{abstract}
RESUMO
Sistemas de Internet das Coisas (IoT) estão em crescente desenvolvimento nos últimos anos. Exemplos de IoT podem ser vistos em todos os ramos da tecnologia como industrial, militar e de monitoramento climático. Mesmo que o mercado esteja cheio de soluções na área, o problema do ponto único de falha ainda persiste em dispositivos responsáveis pela interconexão de redes distintas como gateways IoT. Possíveis soluções na área propõem redundância de gateway IoT, ao se trabalhar com protocolos como o Virtual Routing Redundancy Protocol (VRRP), o que leva à criação de uma redundância na camada de rede do sistema, para prover mais estabilidade e disponibilidade. Porém, a criação de Redes de Sensores sem Fio (RSSF) redundantes também é necessária pois estas estão diretamente conectadas aos gateways IoT. Esse trabalho propõe uma solução para garantir redundância de gateway para uma rede ZigBee em um sistema IoT, também fazendo o uso do protocolo VRRP para redundância de IP.
\end{abstract}

\section{PALAVRAS-CHAVE}

IoT, IoT Gateway, Redundância, ZigBee, VRRP

\section{INTRODUÇÃO}

Redes de Sensores Sem Fio (RSSF) são conjuntos de dispositivos que contém mecanismos capazes de adquirir e repassar dados relativos aos ambientes que as comportam, por meio de um protocolo de comunicação (Akyildiz et al., 2002). Para a implementação de diversos protocolos é interessante se utilizar uma topologia bem definida e que possibilite a conexão de todos os nós com uma entidade central, conhecida como gateway da rede. As RSSF têm papel importante para viabilização de tecnologias e conceitos mais modernos como o de Internet das Coisas (IoT) (Gubbi et al., 2013).

O ZigBee é um protocolo que pode ser usado em redes de sensores sem fio, com pequenos transmissores capazes de compreender o padrão IEEE 802.15.4. As RSSF ZigBee precisam de uma entidade central, chamada coordenador ou gateway ZigBee, que deve ser capaz de receber todos os dados e tomar decisões sobre a rede. Estes, podem também ser incluídos em projetos de IoT para que agreguem opções de protocolos ao sistema, como em Zhu et al. (2010).

Com um dispositivo funcional e capaz de comunicar-se com diferentes RSSF, é possível fazer a interconexão destas, além da comunicação com a internet (Akyildiz et al., 2002). Tendo os recursos de diversas RSSF heterogêneas e a conexão com a rede mundial de computadores, este dispositivo pode se tornar um gateway IoT (Caldas Filho et al., 2017b). Assim, comunicações entre objetos se tornam viáveis e aplicações podem usufruir com mais facilidade dos dados advindos de qualquer protocolo.

As interações entre protocolos diferentes possibilitam uma boa escalabilidade para sistemas IoT. É possível se ver comunicações que envolvem as tecnologias ZigBee e GPRS, em cidades inteligentes como a de Santander, na Espanha, onde milhares de dispositivos se comunicam gerando dados sobre o local como tráfego de veículos, disponibilidade de estacionamento e irrigação de jardins, estes dados podem ser utilizados por usuários finais através da internet (Sanchez et al. 2014).

As redes de comunicações precisam lidar com problemas como quedas em enlaces, que podem ser resolvidos modificando rotas ou utilizando arquiteturas do tipo mesh, para aumentar a escalabilidade e a disponibilidade de acesso (Clouqueur et al., 2002). Contudo, ainda restam situações nas quais é possível ocorrerem problemas em pontos únicos de falha, como gateways. Uma resolução dessa questão é redundância 
em gateways para redes IP, que é feita por meio da implementação de protocolos da classe First Hop Redundancy Protocols (FHRP). Um exemplo é o Virtual Router Redundancy Protocol (VRRP), que será utilizado neste trabalho e é comparado com diversos outros em Pavlik et al. (2014).

A utilização de um protocolo de FHRP para prover redundância de gateway IoT pode ser uma boa solução pois eliminaria problemas de comunicação com a nuvem. Entretanto, para protocolos que atuam em RSSF e exigem um nó central conectado ao gateway IoT, como o ZigBee, a alteração de host no qual o gateway ZigBee se encontra deixaria a rede inteira enviando pacotes para um dispositivo incapaz de repassá-los para um gateway IoT e consequentemente para a internet. Desta maneira, é importante propor algoritmos que possam fazer a redundância de gateway ZigBee para uma rede de sensores.

Este trabalho busca agregar funcionalidades ao gateway semântico proposto em Caldas Filho et al. (2017b). Nele é possível a comunicação em diferentes protocolos, por meio da tradução dos pacotes para uma ontologia que é aceita pelo middleware IoT. Outras funcionalidades deste gateway são o registro e autenticação de dispositivos, a possibilidade de utilização de recursos de dispositivos inteligentes ou de RSSF por usuários finais através do middleware e a tradução de protocolos como ZigBee para uma API REST. Aqui, serão adicionadas funcionalidades de redundância na camada 3 e para a RSSF ZigBee, por meio do VRRP e de um algoritmo proposto respectivamente.

A divisão deste artigo é feita da seguinte maneira: as seções 2 e 3 mostram conceitos e trabalhos relacionados, respectivamente, a seção 4 propõe a implementação do gateway ZigBee com redundância, a seção 5 descreve as simulações e os resultados experimentais obtidos com o gateway proposto, por fim, a seção 6 mostra as conclusões obtidas com a elaboração do gateway, bem como alguns trabalhos futuros.

\section{CONCEITOS RELACIONADOS}

\subsection{Arquitetura IoT}

Baseado no IEEE 802.15.4 e mantido pela ZigBee Alliance, o ZigBee é um protocolo de comunicação que busca conectar dispositivos com baixos recursos. Deste modo, é possível se fazer a comunicação entre nós de uma RSSF necessitando baixas taxas de processamento, pouca bateria e com uma boa escalabilidade. Por outro lado, o protocolo não conta com altas taxas de transmissão como as de outros protocolos também de comunicações sem fio e móveis.

A topologia do ZigBee pode variar e isso proporciona diversas implementações, como mesh, onde dispositivos finais mandam dados para os seus vizinhos, que os repassam até que o dado chegue no nó concentrador ou gateway, ou redes do tipo estrela, onde os dispositivos finais enviam os dados diretamente para o gateway. As três principais classes de dispositivo que compõem a rede são dispositivos finais, roteadores e coordenadores, que atuam como gateways das redes ZigBee. Toda rede deve contém apenas 1 coordenador que atuará fazendo a interconexão da RSSF ZigBee, definindo parâmetros importantes para a mesma, como canal de frequência utilizado e o Personal Area Network Identifier (PAN ID), que diferencia as redes ZigBee. Os roteadores e dispositivos finais podem existir ou não, a diferença entre eles é que roteadores podem se conectar à um ou mais nós ZigBee, enquanto os dispositivos finais estão sempre nas extremidades da rede.

\subsection{ZigBee}

IoT compreende a interconexão entre aplicações, ambientes e dispositivos pela Internet. Conforme destaca Caldas Filho et al. (2017a), a sua arquitetura contém comumente objetos, gateways e middleware. Uma rede IoT tende a manipular um grande volume de dados, sendo assim o middleware e as aplicações são softwares comumente projetados para fazerem proveito da arquitetura em nuvem (Martins et al., 2017).

Os dispositivos possuem o papel de sentir (sensores) e/ou agir (atuadores) sobre o mundo físico como define Ashton et al. (2009) em seu seminal trabalho sobre IoT. Eles podem ser dispositivos com elevado poder de processamento e armazenamento, como smartphones, ou dispositivos com poucos recursos de conectividade, energia, armazenamento e processamento. Os gateways IoT surgiram como os componentes responsáveis por satisfazer a necessidade de interconexão de dispositivos heterogêneos e limitados com o middleware hospedado em nuvem. Nesse sentido, suas principais atribuições são: 1) traduzir a tecnologia e o protocolo de comunicação dos dispositivos para o middleware e 2) indicar a localização do middleware para os dispositivos. Da mesma 
forma, o gateway IoT pode também fazer a conexão entre RSSF com middleware IoT, permitindo que os serviços providos por estas redes façam parte da rede IoT.

Outra arquitetura comum também é feita por meio da computação em neblina, que pode resolver problemas de latência devido ao envio de dados para processamento em máquinas distantes, pertencentes à nuvem (Yannuzzi et al., 2014).

\section{TRABALHOS RELACIONADOS}

Gateways IoT são foco de muita pesquisa e desenvolvimento por se tratar de uma entidade muito importante na interligação de sistemas heterogêneos. Desse modo, algumas soluções já foram propostas e comparadas à nível teórico e prático. Aqui serão mostrados alguns exemplos de trabalhos relacionados à gateway IoT, redundância de gateway IoT e RSSF ZigBee.

Em Caldas Filho et al. (2017b), é proposta uma arquitetura de gateway IoT semântico capaz de se comunicar com os protocolos ZigBee, MQTT, TCP e UDP. A comunicação ocorre por meio de uma padronização que deve ser seguida na troca de mensagens entre os dispositivos e o gateway além das que ocorrem entre gateway e middleware. $\mathrm{O}$ trabalho define muito bem como deve ser o funcionamento interno ao gateway, porém ainda pode ser melhorado com o uso de redundância, tanto para o protocolo IP quanto para de sensores ZigBee que se comunica com o gateway.

Em Scazzoli et al. (2017), os autores propõem uma implementação de um gateway de RSSF ZigBee e IEEE 802.11. O artigo cria um algoritmo que aumenta a disponibilidade de sistemas e obteve com isso ótimos resultados, porém o foco é em sistemas aviônicos e não na disponibilidade de uma RSSF para melhorar a experiência de usuários de um middleware IoT. Para poder prover a redundância na rede ZigBee, os autores contaram com um método desenvolvido e mostrado em Scazzoli et al. (2017). O algoritmo proposto implica a mudança do Personal Area Network Identifier (PAN ID), nos dispositivos pertencentes à rede após a falha de um coordenador. Essa solução se sai melhor do que a solução de reiniciar o dispositivo, contudo em alguns casos o mesmo PAN ID deve ser mantido para possibilitar a autenticidade em sistemas IoT. Uma sugestão de melhoria para este algoritmo é propor que o coordenador ZigBee não mude apenas devido às falhas na RSSF, mas com base em gatilhos advindos de protocolos FHRP, como o VRRP, que será utilizado no desenvolvimento do algoritmo deste artigo.

O trabalho de Ferreira et al. (2014) mostra como uma rede ZigBee pode ser utilizada como apoio a uma rede IP, para prover mais benefícios para usuários finais. Seu foco principal está na arquitetura de um middleware capaz de facilitar o contato entre pessoas e objetos inteligentes, a implementação da RSSF ZigBee é feita com a utilização de mesh, o que já provê uma maior disponibilidade de acesso aos dispositivos. No entanto, o trabalho não visa e não comenta como seria projetada uma solução para quando um dispositivo coordenador da rede ZigBee sofre algum problema.

Outro trabalho com aplicações de redundância de rede aplicado a redes IoT foi feito e apresentado em Ribeiro et al. (2018), onde é elaborada uma solução utilizando um protocolo FHRP para prover redundância no ponto de vista de IP à um gateway IoT. Para isso é feito o uso do IP virtual, fornecido pelo VRRP, assim como será feito aqui. O trabalho implementa uma ótima solução para o aumento da disponibilidade em um gateway IoT, porém fica limitado a dispositivos que se comunicam via protocolo IP. Desta maneira ainda resta aumentar essa disponibilidade para outras redes de sensores que podem estar associadas ao gateway, como RSSF ZigBee. Essa inclusive é uma proposta de trabalho futuro feita pelos autores.

\section{REDE ZIGBEE COM REDUNDÂNCIA DE GATEWAY}

Descreve-se nesta seção a proposta de aplicação de técnicas de redundância a um gateway ZigBee com o objetivo de aumentar a disponibilidade da RSSF. Para a implementação da solução proposta, expandiu-se o Gateway Semântico UIoT, também chamado Gateway UIoT, proposto em Caldas Filho et al. (2017b).

O Gateway UIoT já possuía a capacidade de interagir com dispositivos ZigBee, pelo seu componente ZigBee Handler, bem como com dispositivos nas tecnologias MQTT, Socket TCP e Socket UDP (Caldas Filho et al., 2017b). Para a rede ZigBee, o Gateway UIoT atua como seu em conjunto com um dispositivo configurado como coordenado, de forma que todos os seus dispositivos da RSSF enviam os dados das suas medições para 
o gateway que, por sua vez, os envia ao middleware.

Nesse cenário, qualquer falha que ocorra com o gateway IoT, seus meios de comunicação ou até mesmo no ZigBee Handler pode provocar a indisponibilidade e a perda dos dados enviados pelos dispositivos ZigBee. Assim como em Ribeiro et al. (2018), este trabalho propõe a inclusão de um segundo gateway IoT para atuar como backup do gateway ativo e, caso alguma inoperância seja detectada, assumir o papel de gateway ativo. Porém, além dessa redundância para protocolos baseados em IP, este trabalho propõe que o gateway passivo seja capaz de atuar como coordenador da rede ZigBee caso o gateway ativo falhe.

Para prover a redundância nos protocolos e tecnologias que utilizam IP, configura-se dois gateways IoT em dispositivos separados dentro da mesma rede. Ambos os gateways possuem seu próprio endereço IP, mas também são capazes de responder por um endereço IP virtual dado pelo VRRP, protocolo capaz de fazer a gerência das interfaces de rede em dispositivos que atuam como gateway, gerando um IP virtual e movê-lo entre os aparelhos que suportam seu uso. Para a utilização do VRRP em dispositivos Linux, pode ser feita a aquisição do pacote keepalived, que permite uma fácil implementação do protocolo, podendo fazer uma troca simples e rápida do IP virtual em diferentes máquinas que possuem o software instalado, elencar até diversas máquinas com diferentes prioridades para obtenção do IP virtual (RFC 5798, 2019).

Para prover a redundância para os dispositivos ZigBee pertencentes a RSSF, não há suporte nativo ou ferramenta que faça a reconfiguração automática de um novo coordenador, caso ele se torne indisponível. Assim, foi desenvolvido um algoritmo que realiza a troca de coordenador baseado em um gatilho vindo do VRRP. Além deste, também se faz necessário um algoritmo para que os nós ligados ao coordenador possam se conectar a um novo gateway no caso de troca e assim redirecionar seus pacotes ao destino correto.
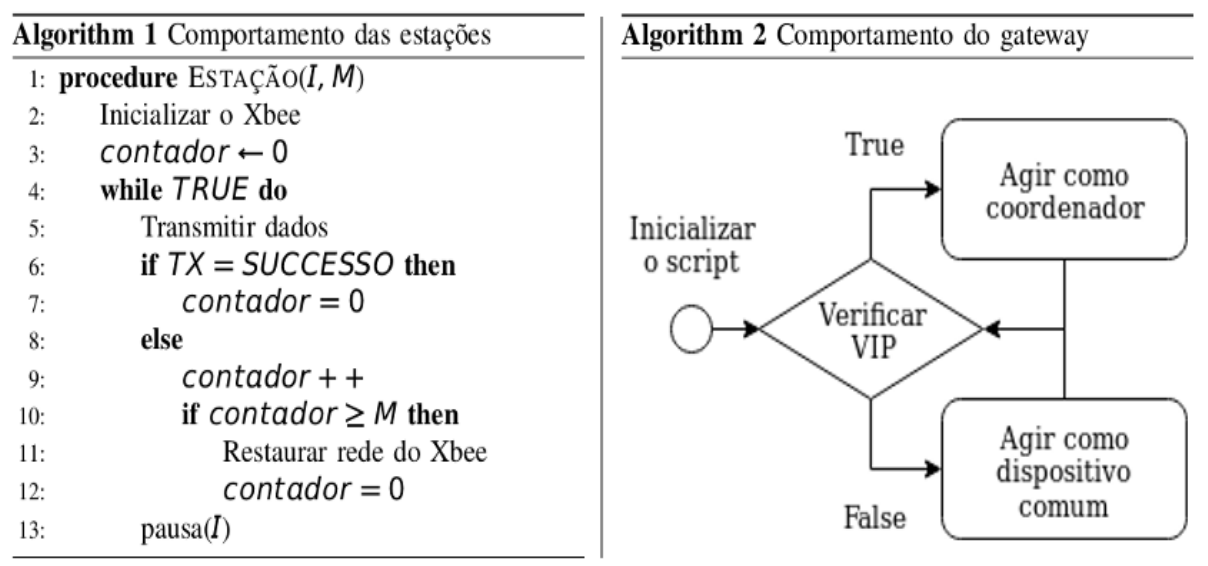

Figura 1. Algoritmos propostos para a realização da redundância proposta

\subsection{Redundância do Gateway ZigBee}

Seguindo o algoritmo 2 apresentado na Figura 1, o processo de alteração do gateway ZigBee deve levar o tempo necessário para o protocolo VRRP alterar o IP virtual entre os Gateways UIoT, principal e redundante, adicionado de um pequeno tempo para configuração dos parâmetros dos dispositivos ZigBee ligados diretamente, aqui foram usados módulos Xbee Series 2. Os parâmetros necessários para alteração são apenas o de Coordinator Enable e de Join Verification, que em dispositivos coordenadores da rede devem ser 1 e 0 , enquanto nos demais deve ser o oposto, 0 e 1.

Na inicialização do procedimento deve ser feita uma verificação para determinar se a instância do Gateway UIoT possui o IP virtual que é provido pelo VRRP. Caso positivo, o Gateway UIoT deve ser tratado como principal e o seu módulo Xbee é configurado como coordenador e será o gateway da rede ZigBee. Caso contrário o módulo deve ser configurado como um roteador ou dispositivo final, como mostra o algoritmo. Tendo feita a inicialização dos módulos corretamente, apenas é necessário fazer uma verificação periódica em busca do IP virtual para que possa ser possível fazer a troca ou a manutenção do estado do módulo ZigBee. O tempo entre verificações pode ser arbitrário, porém tempos muito pequenos podem causar uma sobrecarga no sistema e muito longos podem prolongar demais a troca entre coordenadores. 


\subsection{Redundância dos Nós da Rede ZigBee}

O mecanismo de virtualização do IP do Gateway UIoT provido pelo VRRP permite de forma transparente que os dispositivos, que se usam IP, se comuniquem com o gateway ativo, independentemente de qual gateway esteja ativo na requisição. Entretanto, no ZigBee é necessário que ocorra uma reconfiguração da rede por parte dos dispositivos para que eles se associam ao novo coordenador, o que impede que os dispositivos clientes de um coordenador se comuniquem com o seu substituto de forma automática. Para contornar esse problema, os nós pertencentes à RSSF ZigBee, chamados aqui de estações ZigBee, também devem respeitar um algoritmo bem definido para que trocas de coordenador sejam rapidamente tratadas.

As estações em operação, além de enviarem dados, também devem considerar que, não havendo indicação de recepção deve-se reiniciar suas configurações de redes para que seja possível uma associação ao novo coordenador, processo que é mostrado no algoritmo 1, da Figura 1. Isso também leva a necessidade de um novo registro de cliente e serviços pelo novo Gateway UIoT. O algoritmo proposto deve possibilitar que as estações possam se recuperar automaticamente de falhas ou mudanças de coordenador na RSSF ZigBee, nele 'I' é o intervalo de tempo entre a transmissão de pacotes sucessivos e ' $M$ ' o número máximo de pacotes que podem ser perdidos para se considerar uma mudança de coordenador.

\section{RESULTADOS EXPERIMENTAIS}

Esta seção apresenta a especificação da implementação da proposta, bem como os cenários e os resultados utilizados para validá-la. Para obter os benefícios da solução proposta, serão apresentados os detalhes da implementação da solução proposta, bem como os resultados de tempo de convergência de uma RSSF ZigBee ao se ocorrer uma mudança no coordenador da rede.

\subsection{Dispositivos e Gateways Implementados}

Foi necessário implementar e configurar dispositivos e Gateways UIoT físicos para testar a arquitetura e os algoritmos propostos. Além da construção física, os dispositivos e os gateways foram configurados para executarem os softwares propostos neste trabalho.

Os dispositivos IoT partícipes da RSSF ZigBee normalmente não necessitam de muitos recursos de processamento durante a sua atuação na RSSF. Desta maneira, Arduinos se colocam como grandes aliados à prototipação, ou até mesmo a construção, destes dispositivos. Além disso, existem bibliotecas de código aberto que podem facilitar a sua implementação. Contudo, o Arduino não possui nativamente interface para se comunicar pelo protocolo ZigBee, mas essa capacidade pode ser facilmente provida por meio de um módulo de expansão. Os módulos Xbee Series 2 são dispositivos que podem se comunicar por meio do protocolo ZigBee. Eles são pequenos e requerem baixos requisitos de hardware para seu funcionamento. Desta maneira, favorecem a implementação de redes de sensores ZigBee em diversos ambientes e podem ser utilizados para a comunicação entre dispositivos finais e o gateway UIoT.

O Gateway UIoT é um componente que exige mais recursos computacionais visto que tem importantes funções na rede, de forma que o Arduino não é adequado à sua implementação. Assim, o Raspberry Pi foi escolhido para tal função. Um Raspberry Pi é um dispositivo que conta com um processador, sendo muito mais poderoso que Arduinos em termos de computação. Estes aparelhos contam com diversos benefícios como baixo custo, eficiência energética boa, alta portabilidade e uma boa escala de tamanho. Utilizou-se Raspberry Pi 3 para a implementação dos Gateways UIoT. Também no caso do Raspberry Pi, é necessário acrescentar um módulo extra para dar a conectividade pelo protocolo ZigBee.

\subsection{Cenários}

Para a execução dos cenários propostos nesta seção, os dispositivos foram configurados conforme arquitetura ilustrada na Figura 2: dois gateways IoT com capacidade de coordenar RSSF ZigBee e um dispositivo ZigBee, utilizado para os testes de comunicação via ZigBee. 


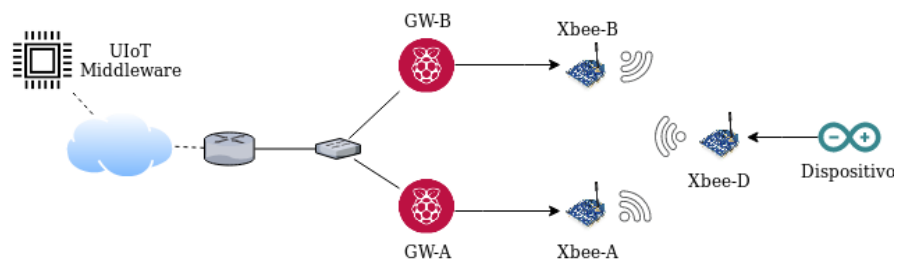

Figura 2. Diagrama da topologia da rede proposta para testes

Os dispositivos ZigBee da RSSF, em funcionamento normal, devem enviar os dados recebidos de sensores para um gateway ZigBee, acoplado ao gateway IoT principal, que serão denominados Xbee-A e GW-A, respectivamente. O GW-A fará a recepção dos dados providos pelo Xbee-A e os passará para processamento local ou envio para a nuvem. Outro gateway IoT, GW-B, ficará como opção de redundância, também com um módulo ZigBee disponível. Levando em conta os tipos de nó presentes numa topologia ZigBee, apenas é necessário fixar GW-A como coordenador da rede.

Em um cenário sem falhas, todas as mensagens enviadas pelo nó, Xbee-D, devem chegar ao GW-A, assim o GW-B fica sempre a espera de uma falha para assumir como gateway principal. O outro cenário possível é o de uma falha que implica a mudança de um Gateway UIoT para outro dispositivo. Essa falha deve ser tratada com base nos algoritmos apresentados, para que assim os dados possam continuar chegando à internet, caso necessário.

Uma vez que as mudanças no gateway ZigBee ocorrem muito rapidamente, por somente dependerem de configurações dos parâmetros indicados na Seção 4, os testes foram feitos apenas para definir o tempo de convergência da RSSF ZigBee após uma falha no enlace. Para facilitar o processo de testes, valores de M e I foram fixados em: $\mathrm{I}=\{1,5,10,30\}$ e $\mathrm{M}=\{1,5,10\}$. Além disso, 30 testes foram feitos para cada combinação de elementos de I e M, resultando em 360 cenários de falha onde em todos a RSSF pode continuar o envio de dados após uma mudança de coordenador.

O tempo de convergência pode ser obtido do tempo total $\mathrm{T}$, entre a recepção do último pacote antes da troca de coordenador e a do primeiro pacote no novo coordenador. Este tempo pode ser dado em função do produto do intervalo de tempo entre a transmissão de pacotes, I, e do número máximo de pacotes que podem ser perdidos, $\mathrm{M}$, adicionado a um tempo de convergência, como mostram as equações 1 e 2 .

$$
\begin{aligned}
& \mathrm{T}(\mathrm{I}, \mathrm{M})=\mathrm{I} . \mathrm{M}+\mathrm{T} \_ \text {Convergencia } \\
& \mathrm{T} \_ \text {Convergencia }=\mathrm{T}(\mathrm{I}, \mathrm{M})-\mathrm{I} . \mathrm{M}
\end{aligned}
$$

\subsection{Tempo de Convergência da RSSF ZigBee}

Após a mudança do coordenador, os nós da rede devem se reiniciar e procurar um novo coordenador, seguindo o algoritmo mostrado na Figura 1. Essa implementação no código do Arduino possui um tratamento em que a reinicialização depende da aceitação máxima de perda de pacotes, $\mathrm{M}$, e do intervalo de tempo entre a transmissão de pacotes, I. A Figura 3, mostra o resultado em segundos, dos testes realizados.

Na Tabela 1, é possível ver o tempo médio, T, da equação 1. Além do dele, é interessante analisar o desvio padrão que deve mostrar se os resultados no teste estão próximos ou distantes do resultado médio. O resultado pode ser considerado bom, pois mesmo os valores mais altos, entre 8 e 9 encontrados para valores de 'I' de 10 e 30, poderiam ser justificados. Esses altos valores ocorrem porque, quando um intervalo grande é testado, o resultado pode variar muito mais, dependendo de quando o coordenador da rede falhar em relação ao intervalo. Por exemplo, em intervalos de 30 segundos se um nó transmitir com sucesso e 1 segundo após a transmissão o coordenador falhar, deve-se esperar 29 segundos para o nó detectar primeira falha na transmissão, mas se o nó transmitir 1 segundo após uma pane os resultados podem ser menores, devido à primeira falha aparecer em apenas 1 segundo e não em 29. Além dos resultados de tempo também é válido se observar uma estimativa da quantidade de pacotes que foram perdidos, apresentado também na Tabela 1, dado pelo tempo T dividido pelo intervalo de transmissão de pacotes I.

Os resultados práticos da equação 2 são mostrados como um histograma na Figura 3. O gráfico mostra que um pequeno intervalo entre a transmissão de pacotes, como 1 segundo, diminui o desempenho da convergência da rede e pode levar até um minuto para convergir, pela implementação feita em $\mathrm{C}++$. Outro ponto é que intervalos maiores, como 10 e 30 segundos, tiveram um tempo de convergência constante, independentemente do valor da perda máxima de pacotes aceitável e mesmo com o desvio padrão mais alto, mostrado na Figura 3. 
Em resumo, a Figura 3 mostra que o tempo de convergência varia em função de 'M' e 'I', para pequenos intervalos de tempo entre pacotes e variar apenas em função de 'I', para o maior intervalos.

Tabela 1. Resultados obtidos com a implementação dos algoritmos propostos

\begin{tabular}{|c|c|c|c|c|c|c|c|c|c|c|}
\hline & & \multicolumn{9}{|c|}{ Número máximo de perda de pacotes } \\
\hline & & \multicolumn{3}{|c|}{ Média (segundos) } & \multicolumn{3}{|c|}{ Desvio Padrão (segundos) } & \multicolumn{3}{|c|}{ Pacotes perdidos } \\
\hline & & 1 & 5 & 10 & 1 & 5 & 10 & 1 & 5 & 10 \\
\hline \multirow{4}{*}{$\begin{array}{c}\text { Intervalo de } \\
\text { tempo entre } \\
\text { transmissão } \\
\text { de pacotes }\end{array}$} & 1 & 24 & 47 & 70 & 0,95 & 0,76 & 1,91 & 24 & 47 & 70 \\
\hline & 5 & 33 & 40 & 66 & 1,87 & 2,55 & 1,91 & 6,6 & 8 & 13,2 \\
\hline & 10 & 28 & 67 & 117,5 & 8,39 & 3,95 & 3,6 & 2,8 & 6,7 & 11,75 \\
\hline & 30 & 57 & 177 & 329,5 & 8,97 & 8,53 & 8,44 & 1,9 & 5,9 & 10,98 \\
\hline
\end{tabular}

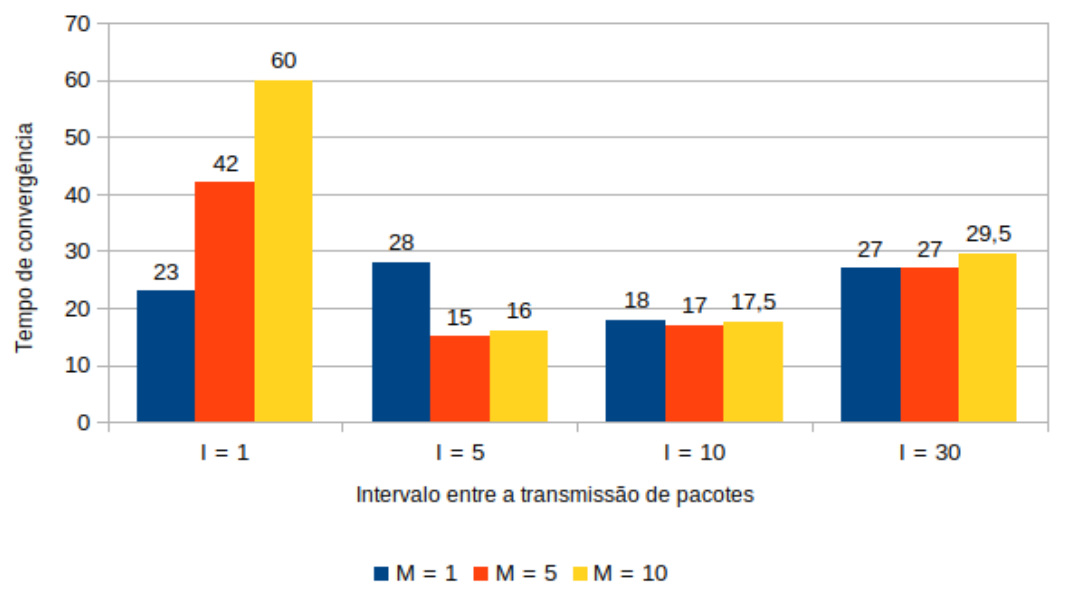

Figura 3. Gráficos dos resultados obtidos experimentalmente para o tempo de convergência mostrado na equação 2

O histograma deixa claro que inundar a rede ZigBee com pacotes a cada segundo prejudica o desempenho dos nós na reinicialização da rede. É importante lembrar que pequenos intervalos entre pacotes não são interessantes quando se lida com sistemas como o ZigBee, devido aos recursos de energia. O ZigBee é conhecido mundialmente como um protocolo de comunicação que requer apenas uma pequena quantidade de energia, mas quanto menor o intervalo de transmissão, maiores os recursos energéticos necessários.

\section{CONCLUSÃO}

A redundância de gateway ZigBee proposta neste artigo, foi feita para prover mais funcionalidades para o gateway UIoT, além de aumentar a disponibilidade para usuários do middleware UIoT. A implementação feita, permite abstrair a necessidade de intervenções humanas no caso de falhas na rede, fazendo com que a comunicação via ZigBee não perca muitos pacotes em caso de pane no gateway principal.

O resultado obtido somente foi possível devido ao uso do VRRP. Este protocolo possibilitou a redundância de IP para os gateways e é a base para a decisão de troca de parâmetro do módulo Xbee associado a um dispositivo que atua como gateway, tornando o dispositivo ZigBee o coordenador da RSSF. O projeto proposto pode ser usado em diversos ramos da tecnologia, desde indústrias e cidades inteligentes até automação residencial, como mostrado na Seção 1.

Durante a pesquisa também foi possível se observar possíveis trabalhos futuros. Melhorias no código e na configuração, podem ser feitos com o foco na obtenção de uma melhor eficiência energética e tempo de convergência dos nós da rede. Além disso, mudanças de configuração e topologias também poderiam prover uma redundância para a própria rede por meio de uma topologia mesh, que é completamente aceitável pelos dispositivos ZigBee utilizados. Outro trabalho futuro seria a expansão das funcionalidades do gateway UIoT para acolher à necessidade de outros protocolos como o LoRa, em crescente uso, adicionando assim mais recursos para o middleware IoT. 


\section{AGRADECIMENTO}

Os autores agradecem o apoio das Agências brasileiras de pesquisa, desenvolvimento e inovação CNPq (Projeto INCT SegCiber 465741/2014-2), CAPES (Projeto FORTE 23038.007604/2014-69) e FAPDF (Projetos UIoT 0193.001366/2016 e SSDDC 0193. 001365/2016), bem como o suporte do Laboratório LATITUDE/UnB (Projeto SDN 23106. 099441/2016-43), a cooperação com o Ministério da Economia (TEDs DIPLA 005/2016 e ENAP 083/2016) e o Gabinete de Segurança Institucional da Presidência da República (TED 002/2017) e à Universidade de Brasília (Edital DEG 05/2019).

\section{REFERÊNCIAS}

Akyildiz et al (2002), Wireless sensor networks: a survey. Computer networks, Vol. 38, No. 4, pp. 393-422.

Ashton et al (2009), That 'internet of things' thing. RFID journal, Vol. 22, No. 7, pp. 97-114.

Caldas Filho et al (2017a), Gerenciamento de serviços IoT com Gateway Semântico, 2017 Conferência Ibero-Americana de Computação Aplicada.

Caldas Filho et al (2017b), Design and Evaluation of a Semantic Gateway Prototype for IoT Networks, 2017. Companion Proceedings of the10th International Conference on Utility and Cloud Computing.

Clouqueur et al (2002), Analysis of span-restorable mesh networks. IEEE journal on selected areas in communications, Vol. 20, No, 4, pp. 810-821Computing.

Ferreira et al (2014), A ubiquitous communication architecture integrating transparent UPnP and REST APIs, International Journal of Embedded Systems, Vol. 6, No. 2-3, pp. 188-197.

Gubbi et al (2013), Internet of Things (IoT): A vision, architectural elements, and future directions. Future generation computer systems, Vol. 29, No. 7, pp. 1645-1660.

Martins et al (2017), Increasing the Dependability of IoT Middleware with Cloud Computing and Microservices. Companion Proceedings of the 10th International Conference on Utility and Cloud Computing.

Pavlik et al (2014), Gateway redundancy protocols. 2014 IEEE 15th International Symposium on Computational Intelligence and Informatics (CINTI), pp. 459-464.

RFC 5798, IETF, acessado em 17 de setembro de 2019, <https://tools.ietf.org/html/rfc5798>

Ribeiro et al (2018), Protocolos de Redundância de Gateway Aplicados em Redes IoT. XXXVI Simpósio Brasileiro de Telecomunicações e Processamento de Sinais (SBrT).

Sanchez et al (2014), SmartSantander: IoT experimentation over a smart city testbed. Computer Networks, Vol. 61, pages $217-238$.

Scazzoli et al (2017a), Recovery in Time-Synchronized Mission Critical ZigBee-Based Wireless Sensor Networks. International Journal of Wireless Information Networks, Vol. 24, No. 3, pp. 268-277.

Scazzoli et al (2017b), A redundant gateway prototype for wireless avionic sensor networks. IEEE 28th Annual International Symposium on Personal, Indoor, and Mobile Radio Communications (PIMRC), pp. 1-7.

Yannuzzi et al (2014), Key ingredients in an IoT recipe: Fog Computing, Cloud computing, and more Fog Computing. 2014 IEEE 19th International Workshop on Computer Aided Modeling and Design of Communication Links and Networks (CAMAD), pp, 325-329.

Zhu et al (2010), Iot gateway: Bridging wireless sensor networks into internet of things. 2010 IEEE/IFIP International Conference on Embedded and Ubiquitous Computing, pp. 347-352. 